\title{
A characterization of some $q$-multiplicative functions
}

\author{
by \\ Jean-Loup Mauclaire (Paris)
}

\section{INTRODUCTION}

1.1. Definition. Let $\mathbb{N}$ be the set of non-negative integers, and let $q>1$ be an integer. To every element $n$ of $\mathbb{N}$, one can associate a unique representation

$$
n=\sum_{k=0}^{\infty} a_{k}(n) q^{k}, \quad 0 \leq a_{k}(n) \leq q-1 .
$$

Following Gelfond [2], a complex-valued arithmetic function $f$ such that $f\left(0 \cdot q^{k}\right)=1$ for all $k \geq 0$ and

$$
f(n)=\prod_{k \geq 0} f\left(a_{k}(n) q^{k}\right)
$$

is called a $q$-multiplicative function.

1.2. Introductory remarks. Since the first investigations of Delange [1], the study of $q$-additive functions, and $q$-multiplicative functions of modulus 1 has been developed by many authors. Apparently, the case of $q$ multiplicative functions not of modulus 1 does not seem to have been so popular, and concerning this topic, we can cite, as recent references, an article of Spilker [6] and another one of Lee [4], both relating to the almostperiodicity of $q$-multiplicative functions. In this article, we shall give some results concerning a class of $q$-multiplicative functions satisfying a growth condition.

\section{RESULTS}

We shall prove the following results:

THEOREM 1. Let $f$ be a non-negative q-multiplicative function. Then (i) \&(ii) $\Leftrightarrow($ iii $) \&($ iv $)$, where

2000 Mathematics Subject Classification: 11A25, 11N64, 11N56.

Key words and phrases: mean-value, $q$-multiplicative functions. 
(i) $0<\limsup _{x \rightarrow \infty} \frac{1}{x} \sum_{n \leq x} f(n)<\infty$,

(ii) if $I(\cdot)$ is the characteristic function of a subset of $\mathbb{N}$ then

$$
\lim _{x \rightarrow \infty} \frac{1}{x} \sum_{0 \leq n<x} I(n)=0 \Rightarrow \lim _{x \rightarrow \infty} \frac{1}{x} \sum_{0 \leq n<x} I(n) f(n)=0,
$$

(iii) $\sum_{r \in \mathbb{N}} \sum_{0 \leq a \leq q-1}\left(1-f\left(a q^{r}\right)\right)^{2}<\infty$,

(iv) $\limsup _{k \rightarrow \infty} \sum_{0 \leq r \leq k} \sum_{0 \leq a \leq q-1}\left(f\left(a q^{r}\right)-1\right)<\infty$.

We also have

THEOREM 2. Let $f$ be a non-negative q-multiplicative function satisfying conditions (i) and (ii) of Theorem 1 . Then, for all $r \geq 0, f(\cdot)^{r}$ satisfies the same conditions.

Now, for $y$ in $\mathbb{N}$, we define a function $F_{y-}(\cdot)$ by

$$
F_{y-}(n)=\left(\prod_{0 \leq k \leq y-1} f\left(a_{k}(n) q^{k}\right)\right)\left(\prod_{0 \leq j \leq y-1} \frac{1}{q} \sum_{0 \leq a \leq q-1} f\left(a q^{j}\right)\right)^{-1} .
$$

We have the following result:

Proposition 3. Let $f$ be a non-negative q-multiplicative function satisfying conditions (i) and (ii) of Theorem 1. Then, given any $\varepsilon>0$, there exists a $Y(\varepsilon)$ in $\mathbb{N}$ such that if $y \geq Y(\varepsilon)$, then

$$
\limsup _{x \rightarrow \infty} \frac{1}{x} \sum_{0 \leq n \leq x}\left|F_{y-}(n)-\frac{f(n)}{\prod_{0 \leq r \leq \log x / \log q} \frac{1}{q} \sum_{0 \leq a \leq q-1} f\left(a q^{r}\right)}\right| \leq \varepsilon,
$$

which implies that

$$
\lim _{x \rightarrow \infty}\left(\frac{1}{x} \sum_{0 \leq n \leq x-1} f(n)\right)\left(\prod_{0 \leq r \leq \log x / \log q} \frac{1}{q} \sum_{0 \leq a \leq q-1} f\left(a q^{r}\right)\right)^{-1}=1 .
$$

Remark 1. Condition (ii) can be replaced, for instance, by: for any $\varepsilon>0$, there exists $\eta>0$ such that, if $I(\cdot)$ is the characteristic function of a subset of $\mathbb{N}$ then

$$
\limsup _{x \rightarrow \infty} \frac{1}{x} \sum_{0 \leq n<x} I(n) \leq \eta \Rightarrow \limsup _{x \rightarrow \infty} \frac{1}{x} \sum_{0 \leq n<x} I(n) f(n) \leq \varepsilon .
$$

The next result completes the first one in the general case. 
Theorem 4. Let $f$ be a complex-valued q-multiplicative function. Define a q-multiplicative function $f^{*}$ of modulus 1 or 0 by

$$
f^{*}(n)= \begin{cases}f(n)|f(n)|^{-1} & \text { if } f(n) \neq 0, \\ 0 & \text { if } f(n)=0 .\end{cases}
$$

Suppose that

(i) $0<\limsup _{x \rightarrow \infty} \frac{1}{x} \sum_{n \leq x}|f(n)|<\infty$,

(ii) if $I(\cdot)$ is the characteristic function of a subset of $\mathbb{N}$ then

$$
\lim _{x \rightarrow \infty} \frac{1}{x} \sum_{0 \leq n<x} I(n)=0 \Rightarrow \lim _{x \rightarrow \infty} \frac{1}{x} \sum_{0 \leq n<x} I(n) f(n)=0 .
$$

Then

$(\mathcal{S})$ the non-negative q-multiplicative function $|f(\cdot)|$ satisfies (ii).

Under conditions (i), (ii) and

(iii) $0<\limsup _{x \rightarrow \infty}\left|\frac{1}{x} \sum_{q^{r} \leq n \leq x} f(n)\right|<\infty \quad$ for some $r \geq 0$,

we have not only $(\mathcal{S})$ but also

$$
\sum_{k \geq 0} \sum_{0 \leq a \leq q-1}\left(1-\operatorname{Re} f^{*}\left(a q^{k}\right)\right)<\infty .
$$

Moreover, $(\mathcal{S}) \Leftrightarrow(\mathrm{i}) \&(\mathrm{ii})$, and $(\mathcal{S}) \&\left(\mathcal{S}^{\prime}\right) \Leftrightarrow(\mathrm{i}) \&(\mathrm{ii}) \&(\mathrm{iii})$.

\section{PROOFS}

3.1. Proof of Theorem 1. The steps of the proof are the following:

1) we remark that there is a natural associated structure of a compact space $Z_{q}$ equipped with a probability measure $\mu$;

2 ) we study the structure of the open sets of this space, and prove that they are disjoint unions of "elementary" components;

3 ) we build a (pre-)measure $\nu$ on these open sets;

4) we remark that it defines a Borel measure, still denoted by $\nu$;

5) this Borel measure is absolutely continuous with respect to $\mu$;

6 ) we give an explicit formula for $d \nu / d \mu$ and get Proposition 3;

7) from classical results of probability theory, we deduce Theorems 1 and 2 .

STEP 1: Compact space associated to a q-multiplicative function. Let $q>1$ be an integer, and $f$ a $q$-multiplicative function. We denote by $Z_{q}$ the compact space $(\mathbb{Z} / q \mathbb{Z})^{\mathbb{N}}$ equipped with the measure $\mu=\bigotimes_{N} \mu_{q}$, where $\mu_{q}$ 
is the uniform measure on the discrete space $\mathbb{Z} / q \mathbb{Z}$. An element $a$ of $Z_{q}$ can be written as $a=\left(a_{0}, a_{1}, \ldots\right), 0 \leq a_{k} \leq q-1, k \geq 0$, and an integer is an element of $Z_{q}$ which has only a finite number of digits different from zero. For $a=\left(a_{0}, a_{1}, \ldots\right) \in Z_{q}$ and $k \geq 0$ we set

$$
x_{k-}(a)=\left\{a_{j}\right\}_{0 \leq j \leq k-1}, \quad x_{k+}(a)=\left\{a_{j}\right\}_{j \geq k} .
$$

These are two sequences of random variables on $Z_{q}$. We have the identity

$$
\prod_{0 \leq j \leq k-1} \frac{1}{q} \sum_{0 \leq a \leq q-1} f\left(a q^{j}\right)=\int_{Z_{q}} f\left(x_{k-}\right) d \mu .
$$

STEP 2: Open sets in $Z_{q}$. We denote by $(a, k(a))$ the arithmetical progression $\left\{a+q^{k(a)} n\right\}_{n \in \mathbb{N}}$, where $a, k(a) \in \mathbb{N}$ satisfy $k(a) \geq \log a / \log q$, and by $I_{a, k(a)}$ its characteristic function. Note that $I_{a, k(a)}$ is the restriction to $\mathbb{N}$ of the characteristic function, still denoted $I_{a, k(a)}$, of the elementary open subset $O_{(a, k(a))}$ of $Z_{q}$ defined by

$$
O_{(a, k(a))}=\left(x_{k(a)-}(a), x_{k(a)+}\left(Z_{q}\right)\right),
$$

and that this function is continuous, which implies that

$$
\lim _{x \rightarrow \infty} \frac{1}{x} \sum_{0 \leq n<x} I_{a, k(a)}(n)=\mu\left(O_{(a, k(a))}\right) .
$$

We have the following lemma:

Lemma 5. Let $O$ be an open set in $Z_{q}$, and $I_{O}$ its characteristic function. Then there exists a subset $A(O)$ of $\mathbb{N}$ such that $I_{O}$ can be written as $I_{O}=$ $\sum_{a \in A(O)} I_{a, k(a)}$, i.e. $O$ can be written as the disjoint union $\bigcup_{a \in A(O)} O_{(a, k(a))}$.

Proof. If $O$ is an open set, then for a given $a$ in $O$, there exists an elementary open set $O_{\left(x_{k(a)-}(a), k(a)\right)}$ such that $O_{\left(x_{k(a)-}(a), k(a)\right)} \subseteq O$. So, $O=$ $\bigcup_{a \in O} O_{\left(x_{k(a)-}(a), k(a)\right)}$. Now, if $O_{\left(x_{k(a)-}(a), k(a)\right)} \cap O_{\left(x_{k(b)-}(b), k(b)\right)} \neq \emptyset$, then one of these two sets is contained in the other. As a consequence, $O$ can be written as a disjoint union $\bigcup_{c \in A(O)} O_{(c, k(c))}$, and so $I_{O}=\sum_{c \in A(O)} I_{c, k(c)}$.

SteP 3: Definition of a measure $\nu$ on the open sets of $Z_{q}$. Given a non-negative $q$-multiplicative function $f$ such that

$$
0<S=\limsup _{x \rightarrow \infty} \frac{1}{x} \sum_{0 \leq n<x} f(n)<\infty,
$$

we can define a measure $\nu$ on the open sets of $Z_{q}$ in the following way.

First, we remark that

$$
0<S^{\prime}=\limsup _{k \rightarrow \infty} \frac{1}{q^{k}} \sum_{0 \leq n \leq q^{k}-1} f(n)<\infty .
$$


For let $x_{i}$ be a sequence such that

Then a fortiori,

$$
\frac{1}{2} S \leq \frac{1}{x_{i}} \sum_{0 \leq n<x_{i}} f(n)
$$

and so

$$
\frac{1}{2} S \leq \frac{1}{x_{i}} \sum_{0 \leq n \leq q^{\log _{q}\left(x_{i}\right)+1}-1} f(n)
$$

$$
\left(\frac{q^{\log _{q}\left(x_{i}\right)+1}}{x_{i}}\right)^{-1}\left(\frac{1}{2} S\right) \leq \frac{1}{q^{k\left(x_{i}\right)+1}} \sum_{0 \leq n \leq q^{k\left(x_{i}\right)+1}-1} f(n) .
$$

Since $\left(q^{\log _{q}\left(x_{i}\right)+1} / x_{i}\right)^{-1} \geq 1 / q$, this shows that there is some $S^{\prime} \geq \frac{1}{2 q} S$, hence $>0$, such that

$$
0<S^{\prime} \leq \limsup _{k \rightarrow \infty} \frac{1}{q^{k}} \sum_{0 \leq n \leq q^{k}-1} f(n)<\infty .
$$

Now, for a given $I_{a, k(a)}$, if $k \geq k(a)$, we have

$$
\begin{array}{rl}
\frac{1}{q^{k}} \sum_{0 \leq n \leq q^{k}-1} & f(n) I_{a, k(a)}(n) \\
= & \frac{f(a)}{\sum_{0 \leq n \leq q^{k(a)}-1} f(n)}\left(\frac{1}{q^{k}} \sum_{0 \leq n \leq q^{k}-1} f(n)\right) \\
= & f(a)\left(\prod_{0 \leq k \leq k(a)-1} \sum_{0 \leq b \leq q-1} f\left(b q^{k}\right)\right)^{-1}\left(\frac{1}{q^{k}} \sum_{0 \leq n \leq q^{k}-1} f(n)\right),
\end{array}
$$

and so we shall define $\nu\left(I_{a, k(a)}\right)$ by

$$
\nu\left(I_{a, k(a)}\right)=\frac{1}{S^{\prime}} \limsup _{k \rightarrow \infty} \frac{1}{q^{k}} \sum_{0 \leq n \leq q^{k}-1} f(n) I_{a, k(a)}(n),
$$

i.e.

$$
\nu\left(I_{a, k(a)}\right)=\frac{1}{S^{\prime}} f(a)\left(\prod_{0 \leq k \leq k(a)-1} \sum_{0 \leq b \leq q-1} f\left(b q^{k}\right)\right)^{-1} \limsup _{k \rightarrow \infty} \frac{1}{q^{k}} \sum_{0 \leq n \leq q^{k}-1} f(n),
$$

which gives

$$
\begin{aligned}
\nu\left(I_{a, k(a)}\right) & =\frac{1}{S^{\prime}} f(a)\left(\prod_{0 \leq k \leq k(a)-1} \sum_{0 \leq b \leq q-1} f\left(b q^{k}\right)\right)^{-1} S^{\prime} \\
& =f(a)\left(\prod_{0 \leq k \leq k(a)-1} \sum_{0 \leq b \leq q-1} f\left(b q^{k}\right)\right)^{-1} .
\end{aligned}
$$

REMARK 2. $\nu$ is well defined due to the very special structure of the open sets of $Z_{q}$. 
Remark 3. By (1), there exists a sequence $K$ of positive integers $k$ such that

$$
\lim _{\substack{k \in K \\ k \rightarrow \infty}} \frac{1}{q^{k}} \sum_{0 \leq n \leq q^{k}-1} f(n)=\limsup _{r \rightarrow \infty} \frac{1}{q^{r}} \sum_{0 \leq n \leq q^{r}-1} f(n) .
$$

We fix such a sequence. The important point in the choice of $K$ is not the mere existence of the limsup, but the fact that the sequence of averages $q^{-k} \sum_{0 \leq n \leq q^{k}-1} f(n), k \in K$, has a limit point not equal to zero. This remark will be useful for the proof of Theorem 4 .

SteP 4: $\nu$ is a Borel measure. We now consider the set $\mathcal{A}$ of complexvalued continuous functions defined on $Z_{q}$ by

$$
\mathcal{A}=\left\{h=\sum_{l_{a} \in L} l_{a} I_{a, k(a)} ; L \text { finite, } l_{a} \in \mathbb{C}\right\} .
$$

This is an algebra of step functions, and we can assume that $I_{a, k(a)} I_{a^{\prime}, k\left(a^{\prime}\right)}$ $=0$ if $(a, k(a)) \neq\left(a^{\prime}, k\left(a^{\prime}\right)\right)$. By the Stone-Weierstrass theorem ([3, p. 101, note 1.a]), this algebra is dense for the uniform topology in the set of complex-valued continuous functions on $Z_{q}$. We define $\nu(h)$ by $\nu(h)=$ $\sum_{l_{a} \in L} l_{a} \nu\left(I_{a, k(a)}\right)$. Note that this definition agrees with the definition of $\nu\left(I_{a, k(a)}\right)$ given above and does not depend on the way $h$ is written, since

$$
\begin{aligned}
\frac{1}{q^{k}} & \sum_{0 \leq n \leq q^{k}-1} f(n) h(n) \\
& =\frac{1}{q^{k}} \sum_{0 \leq n \leq q^{k}-1} f(n) \sum_{l_{a} \in L} l_{a} I_{a, k(a)}(n) \\
& =\left(\sum_{l_{a} \in L} l_{a} f(a)\left(\prod_{0 \leq k \leq k(a)-1} \sum_{0 \leq b \leq q-1} f\left(b q^{k}\right)\right)^{-1}\right) \frac{1}{q^{k}} \sum_{0 \leq n \leq q^{k}-1} f(n),
\end{aligned}
$$

and so

$$
\begin{aligned}
& \frac{1}{S^{\prime}} \lim _{\substack{k \in K \\
k \rightarrow \infty}} \frac{1}{q^{k}} \sum_{0 \leq n \leq q^{k}-1} f(n) h(n) \\
& =\frac{1}{S^{\prime}}\left(\sum_{l_{a} \in L} l_{a} f(a)\left(\prod_{0 \leq k \leq k(a)-1} \sum_{0 \leq b \leq q-1} f\left(b q^{k}\right)\right)^{-1}\right) \lim _{\substack{k \in K \\
k \rightarrow \infty}} \frac{1}{q^{k}} \sum_{0 \leq n \leq q^{k}-1} f(n) \\
& =\sum_{l_{a} \in L} l_{a} f(a)\left(\prod_{0 \leq k \leq k(a)-1} \sum_{0 \leq b \leq q-1} f\left(b q^{k}\right)\right)^{-1} \\
& =\nu(h)=\sum_{l_{a} \in L} l_{a} \nu\left(I_{a, k(a)}\right) .
\end{aligned}
$$


Observe also that $\nu(1)=1$. Now, it is immediate that, given $\varepsilon>0$, if $h, h^{\prime} \in \mathcal{A}$ satisfy $\sup _{t \in Z_{q}}\left|h^{\prime}(t)-h(t)\right| \leq \varepsilon$, then $\left|\nu\left(h^{\prime}-h\right)\right| \leq \varepsilon$, since $h^{\prime}-h$ can be written as $\sum_{l_{a} \in L} l_{a} I_{a, k(a)}$ with $I_{a, k(a)} I_{a^{\prime}, k\left(a^{\prime}\right)}=0$ if $(a, k(a)) \neq\left(a^{\prime}, k\left(a^{\prime}\right)\right)$, and so $\left|l_{a}\right| \leq \varepsilon$. Hence we get

$$
\left|h^{\prime}-h\right|=\sum_{l_{a} \in L}\left|l_{a}\right| I_{a, k(a)} \leq \sum_{l_{a} \in L} \varepsilon I_{a, k(a)},
$$

which gives

$$
\nu\left(\left|h^{\prime}-h\right|\right) \leq \sum_{l_{a} \in L}\left|l_{a}\right| \nu\left(I_{a, k(a)}\right) \leq \varepsilon \sum_{l_{a} \in L} \nu\left(I_{a, k(a)}\right) \leq \varepsilon \nu(1) \leq \varepsilon \cdot 1=\varepsilon .
$$

As a consequence, $\nu$ defines a continuous linear form on the set of complex-valued continuous functions defined on $Z_{q}$. By the Riesz representation theorem $([3$, p. $129,(11.37)])$, this shows that $\nu$ is a Borel measure on $Z_{q}$.

STEP 5: Absolute continuity of $\nu$ with respect to $\mu$. Let $B$ be a Borel subset of $Z_{q}$. Then, given $\varepsilon>0$, there exists an open set $O$ and a compact set $K$ such that $K \subseteq B \subseteq O$ and $\mu(O-K) \leq \varepsilon$. Since $\nu(1)=1$ and $\nu$ is defined on the open sets of $Z_{q}$, we know that $\nu(K)$ can be defined by $\nu(K)=1-\nu\left(Z_{q}-K\right)$, and to prove that $B$ is $\nu$-measurable, using the Lusin criterion $([5$, p. 68 , (vii) $]$ ), it will be sufficient to show that given a sequence $\left\{O_{j}\right\}_{j \in \mathbb{N}^{*}}$ of open sets such that $\lim _{j \rightarrow \infty} \mu\left(O_{j}\right)=0$, we have $\lim _{j \rightarrow \infty} \nu\left(O_{j}\right)=0$.

Assume the contrary, i.e. that there exists a sequence $\left\{O_{j}\right\}_{j \in \mathbb{N}^{*}}$ of open sets such that $\lim _{j \rightarrow \infty} \mu\left(O_{j}\right)=0$ and $\nu\left(O_{j}\right) \geq 2 \lambda>0$ for some $\lambda>0$. Due to the structure of the open sets of $Z_{q}$ described above, any $O_{j}$ can be written as a disjoint union $\bigcup_{a \in A\left(O_{j}\right)} O_{(a, k(a))}$. Since $\nu\left(O_{j}\right)=\sum_{a \in A\left(O_{j}\right)} \nu\left(O_{(a, k(a))}\right)$ and each term of this sum is non-negative, we can find an $\alpha_{j}$ such that the open set $O_{j, \alpha_{j}}=\bigcup_{a \in A\left(O_{j}\right), k(a) \leq \alpha_{j}} O_{(a, k(a))}$ satisfies $\nu\left(O_{j, \alpha_{j}}\right) \geq \lambda$. Note that the characteristic function $I_{j}$ of $O_{j, \alpha_{j}}$ is periodic with period $q^{\alpha_{j}}$ and that $\lim _{j \rightarrow \infty} \mu\left(O_{j, \alpha_{j}}\right)=0$ since from $O_{j, \alpha_{j}} \subseteq O_{j}$, we have $\mu\left(O_{j, \alpha_{j}}\right) \leq \mu\left(O_{j}\right)$, and $\lim _{j \rightarrow \infty} \mu\left(O_{j}\right)=0$.

From now on, to simplify notation, we write $O_{j}$ for $O_{j, \alpha_{j}}$.

Recalling (1), let $X_{j}$ be a sequence of positive integers such that

$$
S^{\prime}=\lim _{j \rightarrow \infty} \frac{1}{q^{X_{j}}} \sum_{0 \leq n \leq q^{X_{j}-1}} f(n),
$$

and moreover, $X_{j}-\alpha_{j}$ and $X_{j+1}-X_{j}$ tend to infinity as $j \rightarrow \infty$. Observe that this implies that $q^{\alpha_{j}}$ divides $q^{X_{j}}$. Then define a subset of $\mathbb{N}$, with characteristic function $I$, by $I(n)=I_{j-1}(n)$ for $q^{X_{j}} \leq n<q^{X_{j+1}}$. 
We will prove that

$$
\lim _{x \rightarrow \infty} \frac{1}{x} \sum_{0 \leq n<x} I(n)=0
$$

Indeed, given $x$, there exists a unique $i$ such that $q^{X_{i}} \leq x<q^{X_{i+1}}$. We have

$$
\begin{aligned}
\sum_{0 \leq n<x} I(n) & =\sum_{0 \leq n<q^{X_{i-1}}} I(n)+\sum_{q^{X_{i-1} \leq n<q^{X_{i}}}} I(n)+\sum_{q^{X_{i} \leq n<x}} I(n) \\
& =\sum_{q^{X_{i-1} \leq n<q^{X_{i-1}}}} I(n)+\sum_{q^{X_{i}}} I_{i-1}(n)+\sum_{q^{X_{i} \leq n<x}} I_{i}(n) .
\end{aligned}
$$

Now,

$$
\begin{aligned}
& \sum_{0 \leq n<q^{X_{i-1}}} I(n) \leq q^{X_{i-1}}, \\
& \sum_{q^{X_{i-1} \leq n<q^{X_{i}}} I_{i-1}(n)} \leq \frac{q^{X_{i}}-q^{X_{i-1}}}{q^{\alpha_{i-1}}} \sum_{0 \leq n \leq q^{\alpha_{i-1}-1}} I_{i-1}(n) \\
&=\left(q^{X_{i}}-q^{X_{i-1}}\right) \mu\left(O_{i-1}\right),
\end{aligned}
$$

since $I_{i-1}$ is a periodic function with period $q^{\alpha_{i-1}}$. Moreover, using the $q^{\alpha_{i-}}$ periodicity of $I_{i}$, we have

$$
\sum_{q^{X_{i} \leq n<x}} I_{i}(n) \leq \sum_{q^{X_{i} \leq n<q^{\alpha_{i}}\left(\left[x / q^{\alpha_{i}}\right]+1\right)}} I_{i}(n)=\left(\left[\frac{x}{q^{\alpha_{i}}}\right]+1-q^{X_{i}-\alpha_{i}}\right) \sum_{0 \leq n<q^{\alpha_{i}}} I_{i}(n) .
$$

Hence

$$
\sum_{q^{X_{i} \leq n<x}} I_{i}(n) \leq\left(\left[\frac{x}{q^{\alpha_{i}}}\right]+1-\frac{q^{X_{i}}}{q^{\alpha_{i}}}\right)\left(q^{\alpha_{i}} \mu\left(O_{i}\right)\right)
$$

and therefore

$$
\sum_{q^{X_{i} \leq n<x}} I_{i}(n) \leq\left(x+q^{\alpha_{i}}-q^{X_{i}}\right) \mu\left(O_{i}\right) \leq x \mu\left(O_{i}\right) .
$$

So, for $x$ such that $q^{X_{i}} \leq x<q^{X_{i+1}}$, we have

$$
\sum_{0 \leq n<x} I(n) \leq q^{X_{i-1}}+\left(q^{X_{i}}-q^{X_{i-1}}\right) \mu\left(O_{i-1}\right)+x \mu\left(O_{i}\right),
$$

which gives

$$
\begin{aligned}
\frac{1}{x} \sum_{0 \leq n<x} I(n) & \leq \frac{q^{X_{i-1}}}{x}+\frac{q^{X_{i}}-q^{X_{i-1}}}{x} \mu\left(O_{i-1}\right)+\mu\left(O_{i}\right) \\
& \leq \frac{q^{X_{i-1}}}{q^{X_{i}}}+\mu\left(O_{i-1}\right)+\mu\left(O_{i}\right)
\end{aligned}
$$

since $q^{X_{i}} \leq x$. But $X_{i}-X_{i-1} \rightarrow \infty$ as $i \rightarrow \infty$, and $\mu\left(O_{j}\right)=o(1)$ as $j \rightarrow \infty$. As a consequence, we get (2). 
We shall now prove that

$$
\limsup _{x \rightarrow \infty} \frac{1}{x} \sum_{0 \leq n<x} f(n) I(n) \geq \lambda S^{\prime}>0 .
$$

We have

$$
\begin{aligned}
\sum_{0 \leq n<q^{X_{j}+1}} f(n) I(n) & =\sum_{0 \leq n<q^{X_{j}}} f(n) I(n)+\sum_{q^{X_{j} \leq n<q^{X_{j+1}}}} f(n) I(n) \\
& =\sum_{0 \leq n<q^{X_{j}}} f(n)\left(I(n)-I_{j}(n)\right)+\sum_{0 \leq n<q^{X_{j+1}}} f(n) I_{j}(n) \\
& \geq \sum_{0 \leq n<q^{X_{j+1}}} f(n) I_{j}(n)-\sum_{0 \leq n<q^{X_{j}}} f(n) .
\end{aligned}
$$

Now, by condition (i) of Theorem 1, we have $\sum_{0 \leq n<q^{X}} f(n)=O\left(q^{X_{j}}\right)$. Moreover,

$$
\begin{aligned}
\sum_{0 \leq n<q^{X_{j+1}}} f(n) I_{j}(n)= & \left(\sum_{0 \leq n<q^{\alpha_{j}}} f(n) I_{j}(n)\right) \sum_{0 \leq n<q^{X_{j+1}-\alpha_{j}}} f\left(q^{\alpha_{j}} n\right) \\
= & \left\{\left(\sum_{0 \leq n<q^{\alpha_{j}}} f(n) I_{j}(n)\right)\left(\sum_{0 \leq n<q^{\alpha_{j}}} f(n)\right)^{-1}\right\} \\
& \times\left\{\left(\sum_{0 \leq n<q^{\alpha_{j}}} f(n)\right)\left(\sum_{0 \leq n<q^{X_{j+1}-\alpha_{j}}} f\left(q^{\alpha_{j}} n\right)\right)\right\} \\
= & \nu\left(O_{j}\right) \sum_{0 \leq n<q^{X_{j+1}}} f(n) .
\end{aligned}
$$

By choice of the $X_{j}$,

$$
\limsup _{x \rightarrow \infty} \frac{1}{x} \sum_{0 \leq n<x} f(n) I(n) \geq \liminf \nu\left(O_{j}\right) \frac{1}{q^{X_{j+1}}} \sum_{0 \leq n<q^{X_{j+1}}} f(n),
$$

and since $\nu\left(O_{j}\right) \geq \lambda$, we get (3). This contradicts hypothesis (ii) of Theorem 1 , and so $\nu$ is absolutely continuous with respect to $\mu$.

STEP 6: Explicit derivative of the measure $\nu$. Since $\nu$ is a probability measure absolutely continuous with respect to $\mu$, the Radon-Nikodym theorem $([3$, p. $144,(12.17)])$ shows that there exists a non-negative integrable function, say $h$, such that if $B$ is a Borel subset of $Z_{q}$, then $\nu(B)=\int_{B} h d \mu$. We have defined on $Z_{q}$ the two sequences of random variables $x_{k-}(a)=$ $\left\{a_{j}\right\}_{0 \leq j \leq k-1}$ and $x_{k+}(a)=\left\{a_{j}\right\}_{j \geq k}$ for $a=\left(a_{0}, a_{1}, \ldots\right) \in Z_{q}$. Now, given some $a$ in $Z_{q}$, we consider the sequence of open subsets $O_{k}$ of $Z_{q}$ defined by $O_{k}=\left(x_{k-}(a), x_{k+}\left(Z_{q}\right)\right)$. Each characteristic function $I_{O_{k}}$ is continuous and since $\mu\left(O_{k}\right)=1 / q^{k}$, we have 


$$
\begin{aligned}
\frac{\nu\left(O_{k}\right)}{\mu\left(O_{k}\right)} & =f\left(x_{k-}(a)\right)\left(\prod_{0 \leq r \leq k-1} \frac{1}{q} \sum_{0 \leq b \leq q-1} f\left(b q^{r}\right)\right)^{-1} \\
& =\frac{1}{\mu\left(O_{k}\right)} \int_{O_{k}} h(t) d \mu(t)=\frac{1}{\mu\left(O_{k}\right)} \int_{Z_{q}} h(t) I_{O_{k}}(t) d \mu(t) \\
& =\int_{x_{k+}\left(Z_{q}\right)} h\left(x_{k-}(a), x_{k+}(t)\right) d \mu\left(x_{k+}(t)\right) .
\end{aligned}
$$

By a direct application of a classical result of Jessen ([7, p. 108]), we find that the quotient (4) converges in $\mathcal{L}^{1}\left(Z_{q}, \mu\right)$ and $\mu$-almost surely to $h$.

Remark 4. As a consequence, we obtain Proposition 3, since by the Cauchy criterion, given any $\varepsilon>0$, there exists a $Y(\varepsilon)$ such that if $z \geq y$ $\geq Y(\varepsilon)$, then

$$
\int_{Z_{q}}\left|\frac{f\left(x_{y-}(t)\right)}{\prod_{0 \leq r \leq y-1} q^{-1} \sum_{0 \leq b \leq q-1} f\left(b q^{r}\right)}-\frac{f\left(x_{z-}(t)\right)}{\prod_{0 \leq r \leq z-1} q^{-1} \sum_{0 \leq b \leq q-1} f\left(b q^{r}\right)}\right| d \mu(t) \leq \varepsilon,
$$

which can be written as

$$
\frac{1}{q^{z}} \sum_{0 \leq n \leq q^{z}-1}\left|\frac{f\left(x_{y-}(n)\right)}{\prod_{0 \leq r \leq y-1} q^{-1} \sum_{0 \leq b \leq q-1} f\left(b q^{r}\right)}-\frac{f(n)}{\prod_{0 \leq r \leq z-1} q^{-1} \sum_{0 \leq b \leq q-1} f\left(b q^{r}\right)}\right| \leq \varepsilon,
$$

which implies immediately that

$$
\lim _{x \rightarrow \infty}\left(\frac{1}{x} \sum_{0 \leq n \leq x-1} f(n)\right)\left(\prod_{0 \leq r \leq \log _{q} x-1} \frac{1}{q} \sum_{0 \leq b \leq q-1} f\left(b q^{r}\right)\right)^{-1}=1 .
$$

STEP 7 (The end!)

STEP 7.1: Consequence of the continuity of $\nu$

Lemma 6. If $\nu$ is continuous, then $1 / 2 \leq f\left(a q^{k}\right) \leq 3 / 2$ except for a finite set of $a q^{k}$, and

$$
\limsup _{k \rightarrow \infty} \sum_{r=0}^{k} \sum_{0 \leq a \leq q-1}\left(1-f\left(a q^{r}\right)\right)^{2}<\infty .
$$

Proof. First of all, we remark that since $f$ satisfies condition (ii) of Theorem 1 , and by (1), we have

$$
\operatorname{card}\left\{(a, k) ; 0 \leq a \leq q-1, k \geq 0, f\left(a q^{k}\right)=0\right\}<\infty .
$$

For we have 


$$
\begin{aligned}
& \frac{1}{q^{k}} \operatorname{card}\left\{n ; 0 \leq n \leq q^{k}-1, f(n) \neq 0\right\} \\
& =\prod_{0 \leq r \leq k-1} \frac{1}{q} \operatorname{card}\left\{(a, r) ; f\left(a q^{r}\right) \neq 0,0 \leq a \leq q-1\right\} \\
& =\prod_{0 \leq r \leq k-1}\left(1-\frac{1}{q} \operatorname{card}\left\{(a, r) ; f\left(a q^{r}\right)=0,0 \leq a \leq q-1\right\}\right),
\end{aligned}
$$

and this is $o(1)$ if

$$
\limsup _{k \rightarrow \infty} \sum_{0 \leq r \leq k-1} \frac{1}{q} \operatorname{card}\left\{(a, r) ; f\left(a q^{r}\right)=0,0 \leq a \leq q-1\right\}=\infty,
$$

which implies that

$$
\limsup _{k \rightarrow \infty} \frac{1}{q^{k}} \sum_{0 \leq n \leq q^{k}-1} f(n)=0,
$$

a contradiction with (1).

As a consequence, there exists some $k$ such that the restriction of $f$ to $q^{k} \mathbb{N}$ is never zero. To simplify notation, we shall assume that $f\left(a q^{k}\right)$ is never zero ab initio.

Now, since the limit of the sequence

$$
f\left(x_{k-}(a)\right)\left(\prod_{0 \leq r \leq k-1} \frac{1}{q} \sum_{0 \leq b \leq q-1} f\left(b q^{r}\right)\right)^{-1}
$$

(see (4)) exists $\mu$-almost surely, applying the three series theorem ([7, p. 88, Corollaire 1]) to the logarithm of this sequence, we deduce that for any $c>0$,

$$
\sum_{\left\{(a, k) ; \mid \log \left(f\left(a q^{k}\right) / q^{-1} \sum_{0 \leq b \leq q-1} f\left(b q^{k}\right) \mid>c\right\}\right.} q^{-1}<\infty,
$$

and since $f\left(0 \cdot q^{r}\right)=1$ for all $r$, this shows that

$$
\left|\log \left(\frac{1}{q} \sum_{0 \leq b \leq q-1} f\left(b q^{k}\right)\right)\right| \leq c
$$

except for a finite number of $k$, and similarly, from

$$
\sum_{\left\{(a, k) ; \mid \log \left(f\left(a q^{k}\right) / q^{-1} \sum_{0 \leq b \leq q-1} f\left(b q^{k}\right) \mid>c\right\}\right.} q^{-1}<\infty,
$$

we conclude that $\left|\log f\left(a q^{k}\right)\right| \leq 2 c$ except for a finite number of $a$ and $k$. Since $c$ can be chosen as small as we want, there exists some $\kappa$ such that for 
$k \geq \kappa$, we have

$$
\frac{1}{2} \leq \frac{1}{q} \sum_{0 \leq b \leq q-1} f\left(b q^{k}\right) \leq \frac{3}{2} \text { and } \quad \frac{1}{2} \leq f\left(a q^{k}\right) \leq \frac{3}{2}
$$

for all $a$. As above, to simplify notation, we shall assume that this holds $a b$ initio.

Now, it is a famous result of Kakutani $([7$, p. 109]) that $\nu$ is absolutely continuous if and only if the product

$$
\prod_{0 \leq k \leq y} \frac{\left(q^{-1} \sum_{0 \leq b \leq q-1} \sqrt{f\left(b q^{k}\right)}\right)^{2}}{q^{-1} \sum_{0 \leq b \leq q-1} f\left(b q^{k}\right)}
$$

tends to a positive limit as $y \rightarrow \infty$. Since it is a product of positive numbers less than or equal to 1 , this is equivalent to

$\sum_{k \geq 0} \frac{1}{q^{-1} \sum_{0 \leq b \leq q-1} f\left(b q^{k}\right)}\left(\frac{1}{q} \sum_{0 \leq b \leq q-1} f\left(b q^{k}\right)-\left(\frac{1}{q} \sum_{0 \leq b \leq q-1} \sqrt{f\left(b q^{k}\right)}\right)^{2}\right)<\infty$, and by (5) it means that

$$
\sum_{k \geq 0}\left(\frac{1}{q} \sum_{0 \leq b \leq q-1} f\left(b q^{k}\right)-\left(\frac{1}{q} \sum_{0 \leq b \leq q-1} \sqrt{f\left(b q^{k}\right)}\right)^{2}\right)<\infty .
$$

By a classical formula of Lagrange, this is exactly

$$
\frac{1}{2 q^{2}} \sum_{k=0}^{\infty} \sum_{0 \leq a, b \leq q-1}\left(\sqrt{f\left(a q^{k}\right)}-\sqrt{f\left(b q^{k}\right)}\right)^{2}<\infty .
$$

Now, since $f\left(0 \cdot q^{k}\right)=1$ for all $k$, this is equivalent to

$$
\sum_{k=0}^{\infty} \sum_{0 \leq a \leq q-1}\left(1-\sqrt{f\left(a q^{k}\right)}\right)^{2}<\infty
$$

and by (5), this can be written as

$$
\sum_{k=0}^{\infty} \sum_{0 \leq a \leq q-1}\left(1-f\left(a q^{k}\right)\right)^{2}<\infty .
$$

STEP 7.2: Proof of Theorem 2. We remark that the statement is evident for $r=0$. Now, if $0<r \leq 1$, it will be sufficient to prove it for $r=1 / 2$. For if

$$
0<\limsup _{x \rightarrow \infty} \frac{1}{x} \sum_{0 \leq n<x} f(n)^{1 / 2}<\infty,
$$

then using the Hölder inequality, for $1 / 2<r<1$ we get

$$
0<\limsup _{x \rightarrow \infty} \frac{1}{x} \sum_{0 \leq n<x} f(n)^{r}<\infty,
$$


and also if $I$ is the characteristic function of a subset of $\mathbb{N}$ then

$$
\lim _{x \rightarrow \infty} \frac{1}{x} \sum_{0 \leq n<x} I(n)=0 \Rightarrow \lim _{x \rightarrow \infty} \frac{1}{x} \sum_{0 \leq n<x} I(n) f(n)^{r}=0 .
$$

So, the conclusion will be satisfied in the range $] 1 / 2,1[\cup\{1\}$, and by iteration, in $\left.] 1 / 2^{2}, 1 / 2[\cup\{1 / 2\} \cup] 1 / 2,1\right]$. The case $r=1 / 2$ will be solved shortly using the Hölder inequality, and so, the conclusion will be satisfied in $\left.\left.\bigcup_{k>0}\right] 1 / 2^{k}, 1\right]$, i.e. in $\left.] 0,1\right]$.

Now, (8) is an immediate consequence of the absolute continuity of $\nu$ with respect to $\mu$, for the product (6) converges to a positive number, say $\mathcal{L}$, as $y \rightarrow \infty$, and so, for $y$ large enough,

$$
\begin{aligned}
2 \mathcal{L}^{-1 / 2} \prod_{0 \leq k \leq y}\left(\frac{1}{q} \sum_{0 \leq b \leq q-1} f\left(b q^{k}\right)\right)^{1 / 2} & \geq \prod_{0 \leq k \leq y} \frac{1}{q} \sum_{0 \leq b \leq q-1} \sqrt{f\left(b q^{k}\right)} \\
& \geq \frac{1}{2} \mathcal{L}^{-1 / 2} \prod_{0 \leq k \leq y}\left(\frac{1}{q} \sum_{0 \leq b \leq q-1} f\left(b q^{k}\right)\right)^{1 / 2}
\end{aligned}
$$

which yields

$$
0<\limsup _{k \rightarrow \infty} \frac{1}{q^{k}} \sum_{0 \leq n \leq q^{k}-1} f(n)^{1 / 2}<\infty .
$$

To obtain the result for $r>1$, it will be sufficient to prove it for the exponent 2. For if it holds for 2, it will hold for all positive powers of 2 , and hence for all $r \geq 1$ by the Hölder inequality. Now, by (5) and (7), we have

$$
\begin{aligned}
& \sum_{k=0}^{\infty} \sum_{0 \leq a, b \leq q-1}\left(f\left(a q^{k}\right)-f\left(b q^{k}\right)\right)^{2} \\
& \quad \leq \sum_{k=0}^{\infty} \sum_{0 \leq a, b \leq q-1}\left(\sqrt{f\left(a q^{k}\right)}-\sqrt{f\left(b q^{k}\right)}\right)^{2}\left(\sqrt{f\left(a q^{k}\right)}+\sqrt{f\left(b q^{k}\right)}\right)^{2} \\
& \quad \leq\left(2 \cdot \frac{3}{2}\right)^{2} \sum_{k=0}^{\infty} \sum_{0 \leq a, b \leq q-1}\left(\sqrt{f\left(a q^{k}\right)}-\sqrt{f\left(b q^{k}\right)}\right)^{2}<\infty .
\end{aligned}
$$

Since, by the Lagrange formula,

$$
\begin{aligned}
\frac{1}{2 q^{2}} \sum_{k=0}^{\infty} \sum_{0 \leq a, b \leq q-1} & \left(f\left(a q^{k}\right)-f\left(b q^{k}\right)\right)^{2} \\
= & \sum_{k \geq 0}\left(\left(\frac{1}{q} \sum_{0 \leq b \leq q-1} f\left(b q^{k}\right)^{2}\right)-\left(\frac{1}{q} \sum_{0 \leq b \leq q-1} f\left(b q^{k}\right)\right)^{2}\right)
\end{aligned}
$$

and since $1 / 2 \leq f\left(b q^{k}\right) \leq 3 / 2$, this gives 


$$
\sum_{k \geq 0} \frac{1}{q^{-1} \sum_{0 \leq b \leq q-1} f\left(b q^{k}\right)^{2}} \cdot \frac{1}{q} \sum_{0 \leq b \leq q-1} f\left(b q^{k}\right)^{2}-\frac{1}{q}\left(\sum_{0 \leq b \leq q-1} f\left(b q^{k}\right)\right)^{2}<\infty,
$$

and so the product (6) converges to a positive limit, say $\mathcal{L}^{\prime}$, as $y \rightarrow \infty$. We can now conclude in the same way as above in the case $r=1 / 2$.

STEP 7.3: End of proof of Theorem 1. First, we remark that

$$
\limsup _{k \rightarrow \infty} \prod_{0 \leq r \leq k} \frac{1}{q} \sum_{0 \leq a \leq q-1} f\left(a q^{r}\right)=\prod_{0 \leq r \leq k}\left(1-\frac{1}{q} \sum_{0 \leq a \leq q-1}\left(1-f\left(a q^{r}\right)\right)\right) .
$$

Now, since

$$
0<S^{\prime}=\limsup _{k \rightarrow \infty} \prod_{0 \leq r \leq k} \frac{1}{q} \sum_{0 \leq a \leq q-1} f\left(a q^{r}\right)<\infty
$$

and logarithm is a continuous increasing function on $] 0, \infty[$, we get

$$
\begin{aligned}
\log \limsup _{k \rightarrow \infty} & \prod_{0 \leq r \leq k}\left(1-\frac{1}{q} \sum_{0 \leq a \leq q-1}\left(1-f\left(a q^{r}\right)\right)\right) \\
& =\limsup _{k \rightarrow \infty} \log \prod_{0 \leq r \leq k}\left(1-\frac{1}{q} \sum_{0 \leq a \leq q-1}\left(1-f\left(a q^{r}\right)\right)\right)=\log S^{\prime}
\end{aligned}
$$

and since $-1 / 2 \leq 1-f\left(a q^{r}\right) \leq 1 / 2$, we obtain $\limsup _{k \rightarrow \infty} \sum_{0 \leq r \leq k} \frac{-1}{q} \sum_{0 \leq a \leq q-1}\left(1-f\left(a q^{r}\right)\right)+O\left(\frac{1}{q}\left(\sum_{0 \leq a \leq q-1}\left(1-f\left(a q^{r}\right)\right)\right)^{2}\right)=\log S^{\prime}$.

Now, we remark that

$$
\frac{1}{q}\left(\sum_{0 \leq a \leq q-1}\left(1-f\left(a q^{r}\right)\right)\right)^{2} \leq \frac{1}{q} \sum_{0 \leq a \leq q-1}\left(1-f\left(a q^{r}\right)\right)^{2}
$$

and since

$$
\sum_{r \in \mathbb{N}} \sum_{0 \leq a \leq q-1}\left(1-f\left(a q^{r}\right)\right)^{2}<\infty
$$

we conclude that

$$
\limsup _{k \rightarrow \infty} \sum_{0 \leq r \leq k} \frac{-1}{q} \sum_{0 \leq a \leq q-1}\left(1-f\left(a q^{r}\right)\right)<\infty,
$$

i.e.

$$
\limsup _{k \rightarrow \infty} \sum_{0 \leq r \leq k} \sum_{0 \leq a \leq q-1}\left(f\left(a q^{r}\right)-1\right)<\infty .
$$

Hence we have shown that conditions (iii) and (iv) of Theorem 1 hold.

Conversely, assuming that (iii) and (iv) hold, we deduce immediately that $-1 / 2 \leq 1-f\left(a q^{r}\right) \leq 1 / 2$ if $r$ is large enough. It is harmless to assume 
that it is so for all $r$. Now, we reverse the argument:

$$
\limsup _{k \rightarrow \infty} \sum_{0 \leq r \leq k} \sum\left(f\left(a q^{r}\right)-1\right)<\infty
$$

implies that

$$
\limsup _{k \rightarrow \infty} \sum_{0 \leq r \leq k} \frac{-1}{q} \sum_{0 \leq a \leq q-1}\left(1-f\left(a q^{r}\right)\right)<\infty
$$

and since $\sum_{r \in \mathbb{N}} \sum_{0 \leq a \leq q-1}\left(1-f\left(a q^{r}\right)\right)^{2}<\infty$, we find that

$$
\limsup _{k \rightarrow \infty} \log \prod_{0 \leq r \leq k}\left(1-\frac{1}{q} \sum_{0 \leq a \leq q-1}\left(1-f\left(a q^{r}\right)\right)\right)<\infty .
$$

Now, since logarithm is a continuous increasing function on $] 0, \infty[$, we have

$$
\begin{aligned}
\limsup _{k \rightarrow \infty} \log \prod_{0 \leq r \leq k}\left(1-\frac{1}{q} \sum_{0 \leq a \leq q-1}\left(1-f\left(a q^{r}\right)\right)\right) \\
=\log \limsup _{k \rightarrow \infty} \prod_{0 \leq r \leq k}\left(1-\frac{1}{q} \sum_{0 \leq a \leq q-1}\left(1-f\left(a q^{r}\right)\right)\right)
\end{aligned}
$$

and so

$$
0<\limsup _{k \rightarrow \infty} \prod_{0 \leq r \leq k} \frac{1}{q} \sum_{0 \leq a \leq q-1} f\left(a q^{r}\right)<\infty .
$$

The same computation as above shows that the product (6) tends to a positive limit as $y \rightarrow \infty$, and so, by the Kakutani Theorem, the sequence of functions

$$
f\left(x_{k-}(\cdot)\right)\left(\prod_{0 \leq r \leq k-1} \frac{1}{q} \sum_{0 \leq b \leq q-1} f\left(b q^{r}\right)\right)^{-1}
$$

converges in $\mathcal{L}^{1}\left(Z_{q}, \mu\right)$. As a consequence, by the Cauchy criterion, given any $\varepsilon>0$, there exists a $Y(\varepsilon)$ such that if $z \geq y \geq Y(\varepsilon)$, we have

$$
\int_{Z_{q}}\left|\frac{f\left(x_{y-}(t)\right)}{\prod_{0 \leq r \leq y-1} q^{-1} \sum_{0 \leq b \leq q-1} f\left(b q^{r}\right)}-\frac{f\left(x_{z-}(t)\right)}{\prod_{0 \leq r \leq z-1} q^{-1} \sum_{0 \leq b \leq q-1} f\left(b q^{r}\right)}\right| d \mu(t) \leq \varepsilon q^{-1},
$$

which can be written as

$$
\frac{1}{q^{z}} \sum_{0 \leq n \leq q^{z}-1}\left|\frac{f\left(x_{y-}(n)\right)}{\prod_{0 \leq r \leq y-1} q^{-1} \sum_{0 \leq b \leq q-1} f\left(b q^{r}\right)}-\frac{f(n)}{\prod_{0 \leq r \leq z-1} q^{-1} \sum_{0 \leq b \leq q-1} f\left(b q^{r}\right)}\right| \leq \varepsilon q^{-1} .
$$

Denoting by $z$ the expression $[\log x / \log q]+1$, if $I(\cdot)$ is the characteristic 
function of a subset of $\mathbb{N}$ and $\lim _{x \rightarrow \infty} x^{-1} \sum_{0 \leq n<x} I(n)=0$, we have

$$
\begin{aligned}
& \frac{1}{x} \sum_{0 \leq n \leq x}\left|\frac{f\left(x_{y-}(n)\right)}{\prod_{0 \leq r \leq y-1} q^{-1} \sum_{0 \leq b \leq q-1} f\left(b q^{r}\right)}-\frac{f(n)}{\prod_{0 \leq r \leq z} q^{-1} \sum_{0 \leq b \leq q-1} f\left(b q^{r}\right)}\right| I(n) \\
& \leq \frac{q^{z}}{x} \cdot \frac{1}{q^{z}} \sum_{0 \leq n \leq q^{z}-1}\left|\frac{f\left(x_{y-}(n)\right)}{\prod_{0 \leq r \leq y-1} q^{-1} \sum_{0 \leq b \leq q-1} f\left(b q^{r}\right)}-\frac{f(n)}{\prod_{0 \leq r \leq z} q^{-1} \sum_{0 \leq b \leq q-1} f\left(b q^{r}\right)}\right| I(n) \\
& \leq q \cdot \frac{1}{q^{z}} \sum_{0 \leq n \leq q^{z}-1}\left|\frac{f\left(x_{y-}(n)\right)}{\prod_{0 \leq r \leq y} q^{-1} \sum_{0 \leq b \leq q-1} f\left(b q^{r}\right)}-\frac{f(n)}{\prod_{0 \leq r \leq z} q^{-1} \sum_{0 \leq b \leq q-1} f\left(b q^{r}\right)}\right| \\
& \leq q \cdot q^{-1} \varepsilon \leq \varepsilon .
\end{aligned}
$$

Now, we remark that

$$
\frac{f\left(x_{y-}(n)\right)}{\prod_{0 \leq r \leq y} q^{-1} \sum_{0 \leq b \leq q-1} f\left(b q^{r}\right)} \leq C(y)<\infty,
$$

and so

$$
\begin{aligned}
& \mid \frac{1}{x} \sum_{0 \leq n \leq x} \frac{f\left(x_{y-}(n)\right)}{\prod_{0 \leq r \leq y} q^{-1} \sum_{0 \leq b \leq q-1} f\left(b q^{r}\right)} I(n) \\
& \quad-\frac{1}{x} \sum_{0 \leq n \leq x} \frac{f(n)}{\prod_{0 \leq r \leq z} q^{-1} \sum_{0 \leq b \leq q-1} f\left(b q^{r}\right)} I(n) \mid \\
& \quad=\left|\frac{1}{\prod_{0 \leq r \leq z} q^{-1} \sum_{0 \leq b \leq q-1} f\left(b q^{r}\right)} \frac{1}{x} \sum_{0 \leq n \leq x} f(n) I(n)+o(1)\right| \quad \text { as } x \rightarrow \infty \\
& \quad \leq \varepsilon,
\end{aligned}
$$

since $x^{-1} C(y) \sum_{0 \leq n \leq x} I(n)=o(1)$ as $x \rightarrow \infty$. Hence

$$
\limsup _{x \rightarrow \infty} \frac{1}{\prod_{0 \leq r \leq z} q^{-1} \sum_{0 \leq b \leq q-1} f\left(b q^{r}\right)} \sum_{0 \leq n \leq x} f(n) I(n) \leq \varepsilon,
$$

which gives

$$
\limsup _{x \rightarrow \infty} \sum_{0 \leq n \leq x} f(n) I(n) \leq \varepsilon \limsup _{x \rightarrow \infty} \prod_{0 \leq r \leq z} \frac{1}{q} \sum_{0 \leq b \leq q-1} f\left(b q^{r}\right) \leq \varepsilon \Lambda .
$$

Hence

$$
\limsup _{x \rightarrow \infty} \sum_{0 \leq n \leq x} f(n) I(n)=0
$$


3.2. Proof of Theorem 4. Most of the arguments given above which rely on classical probability theory apply in this general case of complexvalued $q$-multiplicative functions, and so the details will be given only when necessary.

STEP 1: $(\mathcal{S})$ holds. This is a consequence of the following result:

Proposition 7. Let $f$ be an arithmetical function satisfying the condition

$$
0<S=\limsup _{x \rightarrow \infty} \frac{1}{x} \sum_{0 \leq n<x}|f(n)|<\infty .
$$

Assume that for any sequence $I(n)$ with values 0 or 1 we have

$$
\lim _{x \rightarrow \infty} \frac{1}{x} \sum_{0 \leq n<x} I(n)=0 \Rightarrow \lim _{x \rightarrow \infty}\left|\frac{1}{x} \sum_{0 \leq n<x} I(n) f(n)\right|=0 .
$$

Then also

$$
\lim _{x \rightarrow \infty} \frac{1}{x} \sum_{0 \leq n<x} I(n)=0 \Rightarrow \lim _{x \rightarrow \infty} \frac{1}{x} \sum_{0 \leq n<x} I(n)|f(n)|=0 .
$$

Proof. Let $M$ be a positive integer. We can assume that $I(n)$ takes the value 0 when $f(n)=0$. If $f(n) \not \equiv 0$, we denote by $f^{*}$ the arithmetical function $f \cdot|f|^{-1}$. Now, when $f^{*}$ is of modulus 1 , for integers $k$ in $[0, M-1]$, we define a sequence $I_{k, M}(n)$ with values 0 or 1 by $I_{k, M}(n)=1$ if $\arg f^{*}(n) \in[2 \pi k / M, 2 \pi(k+1) / M[$, and 0 elsewhere. It is clear that $I(n)=$ $\sum_{0 \leq k \leq M-1} I_{k, M}(n)$. Now, we remark that

$$
\begin{aligned}
\frac{1}{x} \sum_{0 \leq n<x} I(n)|f(n)| & =\frac{1}{x} \sum_{0 \leq n<x}\left(\sum_{0 \leq k \leq M-1} I_{k, M}(n)\right)|f(n)| \\
& =\frac{1}{x} \sum_{0 \leq k \leq M-1}\left(\sum_{0 \leq n<x} I_{k, M}(n)|f(n)|\right) \\
& =\frac{1}{x} \sum_{0 \leq k \leq M-1}\left|e^{2 i \pi k / M} \sum_{0 \leq n<x} I_{k, M}(n)\right| f(n) \mid .
\end{aligned}
$$

Observe that

$$
\begin{aligned}
e^{2 i \pi k / M} & \sum_{0 \leq n<x} I_{k, M}(n)|f(n)|=\sum_{0 \leq n<x} I_{k, M}(n)|f(n)| e^{2 i \pi k / M} \\
= & \sum_{0 \leq n<x} I_{k, M}(n)|f(n)|\left(e^{2 i \pi k / M}-f^{*}(n)\right)+\sum_{0 \leq n<x} I_{k, M}(n)|f(n)| f^{*}(n) \\
= & \sum_{0 \leq n<x} I_{k, M}(n)|f(n)|\left(e^{2 i \pi k / M}-f^{*}(n)\right)+\sum_{0 \leq n<x} I_{k, M}(n) f(n) .
\end{aligned}
$$


Hence

$$
\begin{aligned}
& \frac{1}{x} \sum_{0 \leq n<x} I(n)|f(n)| \\
& =\frac{1}{x} \mid \sum_{0 \leq k \leq M-1}\left(\sum_{0 \leq n<x} I_{k, M}(n)|f(n)|\left(e^{2 i \pi k / M}-f^{*}(n)\right)\right. \\
& \left.+\frac{1}{x} \sum_{0 \leq n<x} I_{k, M}(n) f(n)\right) \mid \\
& \leq \frac{1}{x} \sum_{0 \leq k \leq M-1}\left(\sum_{0 \leq n<x} I_{k, M}(n)|f(n)|\left|e^{2 i \pi k / M}-f^{*}(n)\right|\right) \\
& +\frac{1}{x}\left|\sum_{0 \leq n<x} I(n) f(n)\right|,
\end{aligned}
$$

and this can be written as

$$
\begin{aligned}
& \frac{1}{x} \sum_{0 \leq n<x} I(n)|f(n)| \\
& \quad \leq \frac{1}{x} \sum_{0 \leq k \leq M-1}\left(\sum_{0 \leq n<x} I_{k, M}(n)|f(n)|\left|e^{2 i \pi k / M}-f^{*}(n)\right|\right)+o(1), \quad x \rightarrow \infty .
\end{aligned}
$$

Now, we remark that

$$
I_{k, M}(n)|f(n)|\left|e^{2 i \pi k / M}-f^{*}(n)\right|=I_{k, M}(n)|f(n)| O(1 / M)
$$

with the $O$ uniform in $M$, since $\arg f^{*}(n) \in[2 \pi k / M, 2 \pi(k+1) / M[$. This gives

$$
\begin{aligned}
\frac{1}{x} \sum_{0 \leq k \leq M-1}\left(\sum_{0 \leq n<x}\right. & \left.I_{k, M}(n)|f(n)|\left|e^{2 i \pi k / M}-f^{*}(n)\right|\right) \\
& =O\left(\frac{1}{M}\right) \cdot \frac{1}{x} \sum_{0 \leq k \leq M-1}\left(\sum_{0 \leq n<x} I_{k, M}(n)|f(n)|\right) \\
& =O\left(\frac{1}{M}\right) \cdot \frac{1}{x} \sum_{0 \leq n<x}\left(\sum_{0 \leq k \leq M-1} I_{k, M}(n)\right)|f(n)| \\
& =O\left(\frac{1}{M}\right) \cdot \frac{1}{x} \sum_{0 \leq n<x} I(n)|f(n)| \\
& \leq O\left(\frac{1}{M}\right) \cdot \frac{1}{x} \sum_{0 \leq n<x}|f(n)|=O\left(\frac{1}{M}\right) \cdot O(1)=O\left(\frac{1}{M}\right)
\end{aligned}
$$

since by hypothesis, $x^{-1} \sum_{0 \leq n<x}|f(n)|=O(1)$. Hence

$$
\frac{1}{x} \sum_{0 \leq n<x} I(n)|f(n)|=O\left(\frac{1}{M}\right)+o(1), \quad x \rightarrow \infty,
$$


and since $M$ can be as large as we want, we get

$$
\lim _{x \rightarrow \infty} \frac{1}{x} \sum_{0 \leq n<x} I(n)|f(n)|=0 .
$$

STEP 2. This is only a simple remark:

Proposition 8. If for some $r \geq 0$,

$$
0<\limsup _{x \rightarrow \infty}\left|\frac{1}{x} \sum_{q^{r}-1 \leq n \leq x} f(n)\right|<\infty
$$

then

$$
0<\limsup _{k \rightarrow \infty}\left|\frac{1}{q^{k}} \sum_{q^{r}-1 \leq n \leq q^{k}-1} f(n)\right|<\infty .
$$

Proof. First, we may assume that $r=0$, since the shifted function $n \mapsto$ $f\left(q^{r} n\right)$ is $q$-multiplicative. Now, the result is due to the structure of the formula for the summatory function of a $q$-multiplicative function. For if $x$ is a positive integer, written as $x=\sum_{0 \leq r \leq k} a_{r} q^{r}$ with $a_{k} \neq 0$, we have

$$
\begin{aligned}
S_{x}(f)=\sum_{0 \leq n \leq x} f(n)= & \left(\sum_{0 \leq a \leq a_{k}-1} f\left(a q^{k}\right)\right)\left(\prod_{0 \leq j \leq k-1} \sum_{0 \leq a \leq q-1} f\left(a q^{j}\right)\right) \\
& +f\left(a_{k} q^{k}\right) \sum_{0 \leq n \leq x-a_{k} q^{k}} f(n) .
\end{aligned}
$$

This gives

$$
\begin{aligned}
\left|S_{x}(f)\right| \leq & \left(\sum_{0 \leq a \leq a_{k}-1}\left|f\left(a q^{k}\right)\right|\right)\left|\prod_{0 \leq j \leq k-1} \sum_{0 \leq a \leq q-1} f\left(a q^{j}\right)\right| \\
& +\left|f\left(a_{k} q^{k}\right)\right|\left|\sum_{0 \leq n \leq x-a_{k} q^{k}} f(n)\right| .
\end{aligned}
$$

Since $|f(\cdot)|$ satisfies the hypothesis of Theorem 1, the conclusion of Step 7.1 gives

$$
\sum_{k=0}^{\infty} \sum_{0 \leq a \leq q-1}\left(1-\left|f\left(a q^{k}\right)\right|\right)^{2}<\infty
$$

and so

$$
\left|S_{x}(f)\right| \leq a_{k}(1+o(1))\left|\prod_{0 \leq j \leq k-1} \sum_{0 \leq a \leq q-1} f\left(a q^{j}\right)\right|+(1+o(1))\left|\sum_{0 \leq n \leq x-a_{k} q^{k}} f(n)\right| .
$$

Iterating, we find that if

$$
\limsup _{k \rightarrow \infty}\left|\frac{1}{q^{k}} \sum_{0 \leq n \leq q^{k}-1} f(n)\right|=0
$$


then

$$
\limsup _{x \rightarrow \infty}\left|\frac{1}{x} S_{x}(f)\right|=0
$$

which contradicts the hypothesis.

STEP 3. A simple modification of the argument presented in Step 4 of the proof of Theorem 1 leads to the fact that if as above, we define on $Z_{q}$ a sequence of random variables $x_{k-}(a)=\left(a_{j} q^{j}\right)_{0 \leq j \leq k}$ for $a=\left(a_{0}, a_{1}, \ldots\right) \in Z_{q}$, then the sequence of functions $(9)$ converges in $\mathcal{L}^{1}\left(Z_{q}, \mu\right)$ and $\mu$-almost surely to some limit $g$.

SteP 4: $\left(\mathcal{S}^{\prime}\right)$ holds. First, we recall that in Step 7.1 above, we have proved that it is harmless to assume that $f\left(a q^{k}\right)$ is never zero. A consequence is that the limit of the sequence of functions (9), which converges in $\mathcal{L}^{1}\left(Z_{q}, \mu\right)$ and $\mu$-a.s., is positive $\mu$-a.s. For if we denote this limit by $\Phi(\cdot)$, we have $\mu$-a.s.,

$$
\Phi(t)=\prod_{r \geq 0}\left|f\left(a_{k}(t)\right)\right|\left(\frac{1}{q} \sum_{0 \leq b \leq q-1}\left|f\left(b q^{r}\right)\right|\right)^{-1},
$$

and so, $\mu$-a.s.,

$$
\int \Phi(t) d \mu\left(x_{k-}(t)\right)=\prod_{k \leq r}\left|f\left(a_{k}(t)\right)\right|\left(\frac{1}{q} \sum_{0 \leq b \leq q-1}\left|f\left(b q^{r}\right)\right|\right)^{-1} .
$$

A classical result of Jessen ([7, p. 108]) shows that $\int \Phi(t) d \mu\left(x_{k-}(t)\right)$ converges in $\mathcal{L}^{1}\left(Z_{q}, \mu\right)$ and $\mu$-a.s. to $\int \Phi(t) d \mu(t)$, i.e. to 1 . Hence we see that $\prod_{k \leq r}\left|f\left(a_{k}(t)\right)\right|\left(q^{-1} \sum_{0 \leq b \leq q-1}\left|f\left(b q^{r}\right)\right|\right)^{-1}$ tends to $1 \mu$-a.s. as $k \rightarrow \infty$, which implies immediately that $\Phi(t)$ is positive $\mu$-a.s.

Now, since the sequence of functions $(9)$ converges in $\mathcal{L}^{1}\left(Z_{q}, \mu\right)$, we infer that

$$
\begin{aligned}
\int_{Z_{q}} \mid f\left(x_{k-}(a)\right) & \left(\prod_{0 \leq r \leq k-1} \frac{1}{q} \sum_{0 \leq b \leq q-1} f\left(b q^{r}\right)\right)^{-1} \mid d \mu \\
= & \left(\prod_{0 \leq r \leq k} \frac{1}{q} \sum_{0 \leq b \leq q-1}\left|f\left(b q^{r}\right)\right|\right)\left|\prod_{0 \leq r \leq k} \frac{1}{q} \sum_{0 \leq b \leq q-1} f\left(b q^{r}\right)\right|^{-1}
\end{aligned}
$$

has a positive finite limit. This implies that

$$
\begin{aligned}
\frac{f\left(x_{k-}(a)\right)}{\prod_{0 \leq r \leq k-1} q^{-1} \sum_{0 \leq b \leq q-1} f\left(b q^{r}\right)} \cdot \frac{\prod_{0 \leq r \leq k-1} q^{-1} \sum_{0 \leq b \leq q-1}\left|f\left(b q^{r}\right)\right|}{\left|f\left(x_{k-}(a)\right)\right|} \\
\times \frac{\left|\prod_{0 \leq r \leq k-1} q^{-1} \sum_{0 \leq b \leq q-1} f\left(b q^{r}\right)\right|}{\prod_{0 \leq r \leq k-1} q^{-1} \sum_{0 \leq b \leq q-1}\left|f\left(b q^{r}\right)\right|}
\end{aligned}
$$


converges $\mu$-a.s., since each of the three factors of this product does. Since $f\left(x_{k-}(a)\right)=f^{*}\left(x_{k-}(a)\right)\left|f\left(x_{k-}(a)\right)\right|$, this product is equal to $f^{*}\left(x_{k-}(a)\right) \varpi_{k}$, where $\varpi_{k}$ is defined by

$$
\prod_{0 \leq r \leq k-1} \frac{1}{q} \sum_{0 \leq b \leq q-1} f\left(b q^{r}\right)=\bar{\varpi}_{k}\left|\prod_{0 \leq r \leq k-1} \frac{1}{q} \sum_{0 \leq b \leq q-1} f\left(b q^{r}\right)\right| .
$$

So, $\left|\varpi_{k}\right|=1$, and $f^{*}\left(x_{k-}(a)\right) \varpi_{k}$ converges $\mu$-a.s. to limit $F^{*}(a)$; consequently, the symmetrized sequence $f_{k}^{* \mathrm{~s}}(a, b)$ defined by $f^{*}\left(x_{k-}(a)\right) \overline{f^{*}\left(x_{k-}(b)\right)}$ converges $\mu^{2}$-a.s. to $F^{*}(a) \overline{F^{*}(b)}$. Since all these functions have modulus 1 , there exists an open set $O$ such that $\int_{O} F^{*}(a) \overline{F^{*}(b)} d \mu^{2}(a, b) \neq 0$, and due to the structure of the open sets of $Z_{q}$, the same holds for an elementary set $(r, k(r)) \times(s, k(s))$. This implies that

$$
\lim _{k \rightarrow \infty} \int_{(r, k(r)) \times(s, k(s))} f_{k}^{* \mathrm{~s}} d \mu^{2} \neq 0,
$$

and computing the value of this integral shows that there exists some $t$ in $\mathbb{N}$ such that

$$
\lim _{k \rightarrow \infty}\left|\prod_{t \leq r \leq k} \frac{1}{q} \sum_{0 \leq b \leq q-1} f^{*}\left(b q^{r}\right)\right|^{2} \text { exists and is not zero. }
$$

Using the Lagrange identity (for complex numbers), we see immediately that this is equivalent to

$$
\lim _{k \rightarrow \infty} \sum_{k \geq t} \sum_{0 \leq a \leq q-1}\left(1-\operatorname{Re} f^{*}\left(a q^{k}\right)\right)<\infty
$$

and as a consequence,

$$
\lim _{k \rightarrow \infty} \sum_{k \geq 0} \sum_{0 \leq a \leq q-1}\left(1-\operatorname{Re} f^{*}\left(a q^{k}\right)\right)<\infty .
$$

This is assertion $\left(\mathcal{S}^{\prime}\right)$.

STEP 5. It remains to prove that

1) $(\mathcal{S}) \Leftrightarrow(\mathrm{i}) \&(\mathrm{ii})$,

2) $(\mathcal{S}) \&\left(\mathcal{S}^{\prime}\right) \Leftrightarrow($ i $) \&($ ii $) \&($ iii).

The proof of 1 ) is immediate, since if we have $(\mathcal{S})$, we know, by Theorem 1 , that for any $r$ positive,

$$
0<\limsup _{x \rightarrow \infty} \frac{1}{x} \sum_{n \leq x}|f(n)|^{r}<\infty
$$


and as a consequence, if $I(\cdot)$ is the characteristic function of a subset of $\mathbb{N}$ and $\lim _{x \rightarrow \infty} \frac{1}{x} \sum_{0 \leq n<x} I(n)=0$, then

$$
\limsup _{x \rightarrow \infty}\left|\frac{1}{x} \sum_{0 \leq n<x} I(n) f(n)\right| \leq \limsup _{x \rightarrow \infty} \frac{1}{x} \sum_{0 \leq n<x} I(n)|f(n)|=0
$$

by applying the Hölder inequality for some exponent $r>1$.

It remains to prove that if conditions $(\mathcal{S})$ and $\left(\mathcal{S}^{\prime}\right)$ are fulfilled, then (iii) holds true.

Since

$$
\sum_{k \geq 0} \sum_{0 \leq a \leq q-1}\left(1-\operatorname{Re} f^{*}\left(a q^{k}\right)\right)<\infty,
$$

using the Lagrange identity (for complex numbers), we deduce that there exists some $t$ in $\mathbb{N}$ such that (10) holds. This implies that the sequence of functions $F_{y-}^{*}(x)$ defined on $Z_{q}$ by

$$
F_{y-}^{*}(x)=\left(\prod_{t \leq k \leq y} f^{*}\left(a_{k}(x) q^{k}\right)\right)\left(\prod_{t \leq j \leq y} \frac{1}{q} \sum_{0 \leq a \leq q-1} f^{*}\left(a q^{j}\right)\right)^{-1}
$$

is a bounded martingale convergent in $\mathcal{L}^{\infty}\left(Z_{q}, d \mu\right)$. Similarly, the sequence of functions $F_{y-}(x)$ defined on $Z_{q}$ by

$$
F_{y-}(x)=\left(\prod_{t \leq k \leq y}\left|f\left(a_{k}(x) q^{k}\right)\right|\right)\left(\prod_{t \leq j \leq y} \frac{1}{q} \sum_{0 \leq a \leq q-1}\left|f\left(a q^{j}\right)\right|\right)^{-1}
$$

is a martingale convergent in $\mathcal{L}^{1}\left(Z_{q}, d \mu\right)$.

Hence the sequence $F_{y-}^{*}(x) F_{y-}(x)$ converges in $\mathcal{L}^{1}\left(Z_{q}, d \mu\right)$. Now, since

$$
\begin{aligned}
\lim _{y \rightarrow \infty} \int \mid F_{y-}^{*}(x) F_{y-} & (x) \mid d \mu(x) \\
& =\lim _{y \rightarrow \infty} \int F_{y-}(x)\left(\prod_{t \leq j \leq y}\left|\frac{1}{q} \sum_{0 \leq a \leq q-1} f^{*}\left(a q^{j}\right)\right|\right)^{-1} d \mu(x) \\
& =\lim _{y \rightarrow \infty}\left(\prod_{t \leq j \leq y}\left|\frac{1}{q} \sum_{0 \leq a \leq q-1} f^{*}\left(a q^{j}\right)\right|\right)^{-1} \neq 0,
\end{aligned}
$$

there exists an open set $O$ such that

$$
\lim _{y \rightarrow \infty} \int_{O} F_{y-}^{*}(x) F_{y-}(x) d \mu(x) \neq 0,
$$

and so there exists an elementary set $O_{(a, k(a))}$ such that

$$
\lim _{y \rightarrow \infty} \int_{O_{(a, k(a))}} F_{y^{-}}^{*}(x) F_{y-}(x) d \mu(x) \neq 0 .
$$


This implies that the limit of the product

$$
\begin{aligned}
\left(\prod_{k(a) \leq k \leq y} \frac{1}{q} \sum_{0 \leq a \leq q-1} f\left(a_{k}(x) q^{k}\right)\right) & \cdot\left(\prod_{k(a) \leq j \leq y} \frac{1}{q} \sum_{0 \leq a \leq q-1} f^{*}\left(a q^{j}\right)\right)^{-1} \\
& \times\left(\prod_{k(a) \leq j \leq y} \frac{1}{q} \sum_{0 \leq a \leq q-1}\left|f\left(a q^{j}\right)\right|\right)^{-1}
\end{aligned}
$$

exists and is not zero, and a fortiori, the limit of

$$
\begin{aligned}
\left|\prod_{k(a) \leq k \leq y} \frac{1}{q} \sum_{0 \leq a \leq q-1} f\left(a_{k}(x) q^{k}\right)\right| \cdot \mid & \prod_{k(a) \leq j \leq y} \frac{1}{q} \sum_{0 \leq a \leq q-1} f^{*}\left(a q^{j}\right) \mid \\
& \times\left(\prod_{k(a) \leq j \leq y} \frac{1}{q} \sum_{0 \leq a \leq q-1}\left|f\left(a q^{j}\right)\right|\right)^{-1}
\end{aligned}
$$

exists and is not zero. Now, since

$$
\lim _{y \rightarrow \infty}\left|\prod_{k(a) \leq j \leq y} \frac{1}{q} \sum_{0 \leq a \leq q-1} f^{*}\left(a q^{j}\right)\right|
$$

exists and is not zero, and

$$
0<\limsup _{y \rightarrow \infty} \prod_{k(a) \leq j \leq y} \frac{1}{q} \sum_{0 \leq a \leq q-1}\left|f\left(a q^{j}\right)\right|<\infty,
$$

we get

$$
0<\limsup _{y \rightarrow \infty}\left|\prod_{k(a) \leq k \leq y} \frac{1}{q} \sum_{0 \leq a \leq q-1} f\left(a_{k}(x) q^{k}\right)\right|<\infty,
$$

and so there exists some $r \geq 0$ such that

$$
0<\limsup _{x \rightarrow \infty}\left|\frac{1}{x} \sum_{q^{r} \leq n \leq x} f(n)\right|<\infty .
$$

\section{References}

[1] H. Delange, Sur les fonctions q-additives ou q-multiplicatives, Acta Arith. 21 (1972), 285-298.

[2] A. O. Gelfond, Sur les nombres qui ont des propriétés additives et multiplicatives données, ibid. 13 (1968), 259-265 .

[3] E. Hewitt and K. Ross, Abstract Harmonic Analysis I, Springer, 1963.

[4] Y.-W. Lee, Characterization of almost-periodic q-multiplicative functions, Ann. Univ. Sci. Budapest. Eötvos Lorand Sect. Comp. 22 (2003), 396-403.

[5] P. Malliavin, Intégration et probabilité. Analyse de Fourier et analyse spectrale, Masson, Paris, 1982. 
[6] J. Spilker, Almost-periodicity of g-additive and g-multiplicative functions, in: Analytic and Probabilistic Methods in Number Theory, A. Dubickas et al. (eds.), TEV, Vilnius, 2002, 256-264.

[7] A. Tortrat, Calcul des probabilités et introduction aux processus aléatoires, Masson, Paris, 1971.

Théorie des nombres

Institut de mathématiques (UMR 75867 du CNRS)

Université Pierre et Marie Curie

175 rue du Chevaleret, Plateau 7D

F-75013 Paris, France

E-mail: mauclai@ccr.jussieu.fr

Received on 5.2.2004

and in revised form on 10.2.2005 